\title{
Improving Safe and Effective Use of Drugs in Pregnancy and Lactation: Workshop Summary
}

\author{
Laura E. Riley, $\mathrm{MD}^{1} \quad$ Alison G. Cahill, MD, $\mathrm{MSCl}^{2} \quad$ Richard Beigi, MD, MSc ${ }^{3}$ Renate Savich, $\mathrm{MD}^{4}$ \\ George Saade, $\mathrm{MD}^{5}$
}

1 Division of Maternal Fetal Medicine, Department of Obstetrics and Gynecology, Massachusetts General Hospital, Harvard Medical School, Boston, Massachusetts

2 Department of Obstetrics and Gynecology, Washington University, St. Louis, Missouri

${ }^{3}$ Department of Obstetrics, Gynecology, and Reproductive Sciences, University of Pittsburgh Medical Center, Magee-Women's Hospital, Pittsburgh, Pennsylvania

${ }^{4}$ Division of Newborn Medicine and Neonatal Intensive Care Unit, University of Mississippi Medical Center, Jackson, Mississippi

${ }^{5}$ Department of Obstetrics and Gynecology, University of Texas Medical Branch at Galveston, Galveston, Texas

\begin{abstract}
Address for correspondence Laura E. Riley, MD, Division of Maternal Fetal Medicine, Massachusetts General Hospital, 55 Fruit Street, Founders 420, Boston, MA 02114 (e-mail: Iriley@partners.org).
\end{abstract}

Am J Perinatol 2017;34:826-832.

\begin{abstract}
Keywords

- drug safety

- pregnancy

- lactation

- pharmacokinetics

- drug surveillance

In February 2015, given high rates of use of medications by pregnant women and the relative lack of data on safety and efficacy of many drugs utilized in pregnancy, the Eunice Kennedy Shriver National Institute of Child Health and Human Development (NICHD), the Society for Maternal-Fetal Medicine (SMFM), the American College of Obstetricians and Gynecologists (ACOG), and the American Academy of Pediatrics (AAP) convened a group of experts to review the "current" state of the clinical care and science regarding medication use during the perinatal period. The expert panel chose select medications to demonstrate what existing safety and efficacy data may be available for clinicians and patients when making decisions about use in pregnancy or lactation. Furthermore, these example medications also provided opportunities to highlight where data are lacking, thus forming a list of research gaps. Last, after reviewing the existing vaccine safety surveillance system as well as the legislative history surrounding the use of drugs for pediatric diseases, the expert panel made specific recommendations concerning policy efforts to stimulate more research and regulatory attention on drugs for pregnant and lactating women.
\end{abstract}

Although a large number of pregnant and lactating women receive prescription drugs, over-the-counter (OTC) medications, and supplements, many knowledge gaps persist concerning the safety and efficacy of these therapies. Consequently, there is wide variability in the advice women receive concerning medication use during pregnancy and lactation. To address this, the Eunice Kennedy Shriver National Institute of Child Health and Human Development (NICHD), the Society for Maternal-Fetal
Medicine (SMFM), the American College of Obstetricians and Gynecologists (ACOG), and the American Academy of Pediatrics (AAP) convened a group of experts to a workshop to review the "current" state of the clinical care and science regarding medication use during the perinatal period. These groups were also charged with suggesting guidance for practitioners as well as identification of research gaps. This manuscript provides a summary of the opinions provided by the expert panel. received

November 7, 2016 accepted after revision December 27, 2016 published online January 31, 2017
DOI https://doi.org/ 10.1055/s-0037-1598070. ISSN $0735-1631$
Copyright $\odot 2017$ by Thieme Medical Publishers, Inc., 333 Seventh Avenue, New York, NY 10001, USA. Tel: +1(212) 584-4662.

\section{License terms}

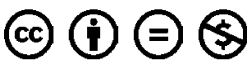


The Centers for Disease Control and Prevention (CDC) reports that the diagnosis of prepregnancy chronic health conditions are on the rise in the United States. ${ }^{1-3}$ These conditions include cardiovascular disease, metabolic disorders, mental health disorders, and respiratory diseases. ${ }^{4-7}$ These trends could be the result of improved modalities for diagnosing and treatments. ${ }^{8-10}$ Because most interventions to manage these conditions employ the use of pharmacologic products that have not been well studied in pregnant and lactating women, the true risks and benefits of such interventions remain speculative. Furthermore, Mitchell et al performed a retrospective study of more than 19,000 women from two case control studies that enrolled patients from 1976 to 2008, and reported that approximately $90 \%$ of women were taking at least one medication, either prescription or OTC, during their pregnancy. ${ }^{11}$ About 7 in 10 women were taking one or more prescription medications during their pregnancy with almost one-third of women using four medications. ${ }^{11}$ In addition, medication use in the first trimester increased by $62.5 \%$ over the study period. The most dramatic increase in prescription medication seen in this study was women who were exposed to antidepressants from 1988 to 1990 (<1\%) and the striking increase and peak at $7.5 \%$ during the study period of 2003 to $2008 .^{11}$ According to Werler et al, two case-control studies demonstrated that acetaminophen was used at least $65 \%$ of the time, whereas ibuprofen and pseudoephedrine exposure during pregnancy occurred in approximately one in six women. ${ }^{12}$ There have been very few studies examining the risks of pregnancy and fetal outcomes after exposure to OTC medications as well as prescription drugs.

Professional entities including SMFM, ACOG, and AAP strongly advocate that women breast-feed their infants because of its numerous maternal and neonatal health benefits. Breast-feeding rates have increased approximately $2 \%$ per year between 1996 and 2001. ${ }^{13,14}$ Stultz et al studied 46 women who provided medication history during their pregnancy and while they breast-fed their infants. ${ }^{15}$ In this cohort, there was a significant increase in both prescription and OTC medication use during lactation compared with when they were pregnant. More than $70 \%$ of women were taking medications that were potentially unsafe, or had unknown safety profiles. Anderson et al evaluated 100 articles between 1966 and 2002 to examine possible adverse outcomes in neonates due to their exposure to maternal medication(s) during breast-feeding. ${ }^{16}$ These authors reported that $47 \%$ of adverse outcomes in the neonates as "possibly" linked to medication exposure during breast-feeding; 53\% as "probably" linked and no infant-related deaths were associated with medications. ${ }^{16}$ However, there is little research focusing on understanding the quantity of medication and their chemical effects on neonates during breast-feeding.

Only $5 \%$ of the 213 new pharmaceuticals approved by the Food and Drug Administration (FDA) between 2003 and 2012 contained human data in their pregnancy section; almost one-half did not have any safety information concerning the medication use for lactating women. ${ }^{17}$ Because of the lack of studies on specific drugs in pregnancy and lactation, the efficacy and dosing data are generally extrapolated from studies from adult subjects (men and nonpregnant women) and safety data are collated from the FDA-mandated postmarket surveillance. Such data often come from the use of pregnancy-exposure registries that, while useful, are greatly limited by inherent design biases.

During the workshop, the expert panel focused on the "current" state of practice and reviewed available data on select drugs in pregnancy. The group discussed what providers optimally should know about the pharmacokinetics of drugs particularly given the physiologic changes that occur in the course of pregnancy and postpartum, as well as the time of greatest vulnerability in terms of drug effects on the fetus and newborn. There was evidence presented about the interaction of drugs and breast milk, indicating that some assumptions could be made about the efficiency with which certain drugs may cross into breast milk and potentially pose further exposure risks to the neonate. Potential lessons learned from the pediatric experience with enrolling children in drug trials to enhance knowledge in this unique population and the robust safety systems in place to monitor vaccines were reviewed. The presentations and discussions from the 2-day workshop are summarized here.

\section{Pharmacokinetics of Drugs in Pregnancy}

An extensive discussion of these pharmacokinetic principles were reported at the workshop and are detailed in a subsequent publication by Feghali et al. ${ }^{18}$ In brief, the pharmacokinetics of a given drug describes the processing of the drug through the body and incorporates several components, including the drug's absorption, distribution, metabolism, elimination, and transport. Usually it is the bioavailability of drug, the amount of drug that is able to target the tissue, which is critical in determining how efficacious the drug will be. This depends on a combination of factors including properties of the drug as well as properties of the host. For example, the bioavailability of orally administered medications is affected by absorption across the intestinal epithelium and by first-pass metabolism in the intestine and liver. All these factors are more variable in pregnancy because the stomach $\mathrm{pH}$, transit time, metabolism, uptake, and efflux transport processes are altered during pregnancy, thereby potentially changing the drug's bioavailability. Conversely, the uptake of the same drug given intravenously or intramuscularly may be less variable than when orally administered, but it remains different in the pregnant state compared with the nonpregnant state in which there is increased cardiac output and increased intestinal blood flow leading to increased overall absorption. In addition to these changes, drug distribution is different in pregnancy in which the distribution of a given drug is altered depending on tissue perfusion, binding, lipid solubility, and plasma protein binding. Again, the expanded plasma volume experienced during pregnancy and the fact that a proportion of a compound may cross the placenta may influence hydrophilic drugs causing lower than anticipated plasma concentrations in the pregnant woman. Similarly, with lipophilic drugs, the expanded maternal body fat during pregnancy may alter the plasma 
concentration and resulting efficacy of these medications. Drug metabolism, the chemical process that converts the drug into its active form, is also heavily influenced by the physiologic changes that occur in pregnancy and certainly influence the activity of a given drug. Drug elimination is another critical component to the bioavailability of that agent. For example, although the glomerular filtration rate is 50\% higher in pregnant than in nonpregnant states and is relatively stable throughout gestation, drugs that are cleared by the kidney act variably, likely due to changes in renal tubular transport. Finally, an area of great importance is drug transport. The fetal-placental compartment and the transport proteins within it greatly influence the amount, timing, and degree of drug exposure for the developing fetus, and how this is different throughout gestation is at least in part related to the variable expression of fetal damage seen with some medications.

\section{Fetal Development and Vulnerability}

Human embryogenesis, the beginning stages of cell division and cell differentiation of the human embryo, depends on the stem cells ability to divide, migrate, and specialize. ${ }^{17}$ This intricate process results in the susceptibility and risk of teratogenicity. The teratogenic period can be particularly severe from day 31 after the last menstrual period in a 28 day cycle to 71 days from the last period. ${ }^{19}$ In this timeframe, teratogens have the potential to influence malformations that are noticeable at birth and exposure to teratogens prior to day 31 may produce an "all-organ effect" in which the conceptus does not survive or survives with anomalies. It is also possible that the fetus survives without anomalies due to the regeneration of any damaged tissue. ${ }^{19}$ In addition to the developmental susceptibility, it is important to note that functional maturation begins in utero and continues into the postnatal period and also remains a time of potential harm. There is differential susceptibility by trimester of pregnancy and organ system. There are several examples where specific fetal malformations are caused by exposure to medications during a critical time in pregnancy such as thalidomide, angiotension-converting enzyme inhibitors, and certain antiepileptic medications. ${ }^{20-22}$

The evaluation of the teratogenic risk of a given drug is complex. Risk assessment is ultimately based on animal, human, and pharmacologic data when available, and scientific evidence of teratogenicity relies on nonclinical data, drug class characteristics, biologic plausibility of exposure, impact of maternal disease or condition, and human data. Animal toxicology studies are designed specifically to identify hazards and potential toxic effects on targeted organs. These animal studies allow assessment of potential hazards that cannot be assessed in human clinical trials; however, they are limited in their applicability to humans. Some animal studies will include data on male and female fertility, parturition, and lactation. There is also an assessment of developmental toxicity, including mortality, dysmorphogenesis, alterations in growth, and functional toxicity. Once the animal studies are reviewed, there is a "data integration process" that then considers several factors, including, but not limited to, dose response, rarity of adverse events, relative exposure, and cross species concordance. However, the significant increase in medications used during pregnancy far outpaces the body of knowledge for effects on the fetus.

\section{Transfer of Drug in Human Breast Milk}

There is limited information on most drugs used by breastfeeding women, which poses challenges for women and their providers. Consequently, many providers will recommend stopping a medication based on theoretical concerns about its safety for the neonate, ${ }^{13}$ and this may lead to inadequately treated maternal disease. Information needed to make decisions about medications and breast-feeding includes knowledge of the need for the drug, the excretion of the drug into breast milk, the extent of oral absorption of the drug by the neonate, the potential adverse effects on the neonate, and the potential effect of the drug on lactation..$^{23,24}$ In addition, the infant's age is relevant and should be considered as adverse events are rarer in infants older than 6 months. As in pregnancy, the biochemical properties of a given drug are important elements such as drug ionization, molecular weight, volume of distribution, lipid solubility, and protein binding, and they may influence how much of the drug is excreted into breast milk. Furthermore, the half-life of the drug and its oral bioavailability will influence how much is absorbed by the neonate. ${ }^{25,26}$ One example of these complexities include emerging data demonstrating that there are specific patient genotypes that influence the toxicity of given drugs. Stover and Davis suggest that this area of pharmacogenomics holds promise in understanding how neonates with in utero exposure to opioids may have different withdrawal patterns based on specific polymorphisms. ${ }^{27}$

The main recommendations from the workshop were focused in the following areas: clinical care recommendations, research gaps, and policy-related considerations.

\section{The Expert Panel's Recommendations for Clinical Care}

Clinicians and patients must make decisions incorporating the available data on the maternal, fetal, and neonatal impact of disease; drug pharmacokinetics; maternal and neonatal safety; limitations of the data sources; and guidelines from scientific bodies if they exist. Panelists used examples of commonly used drugs such as acetaminophen, pseudoephedrine, and antihypertensive agents to illustrate the risk-benefit analysis undertaken to inform clinical practice. ${ }^{28-30}$

- When clinicians utilize any drug or counsel women about OTC medications, there needs to be a risk-benefit analysis, and when there are multiple options, the drug with the most favorable benefit: risk ratio is utilized when considering the health of both the mother and her baby.

- There should be readily accessible information on drugs, which addresses the critical components needed to make clinical decisions for clinicians to apply this risk-benefit analysis. In the last 30 years, providers have relied heavily on the FDA's pregnancy labeling that classified drugs as A, 
B, C, D, and X. However, these categories were vague and often misleading. As of June of 2015, all new medications approved by the FDA have a new pregnancy label with a narrative section that includes a risk summary, clinical considerations, and supporting data. A section on lactation provides data on the neonatal effects of the drug. In addition, the existence of drug registries is displayed. While the new label should represent a vast improvement in providing useful information, most drugs will not have been studied in pregnancy or during lactation, which potentially leaves this new label just as ambiguous. Furthermore, this labeling change pertains to drugs submitted to FDA review after June 2015, and the schedule for revisions of old medications is unknown at this time.

- Other resources for information on drug safety and dosing in pregnancy and lactation include REPROTOX, TERIS, LactMed, and specific drug registries (up-to-date listing can be found on the FDA Web site). Patient-specific education materials for select commonly used medications are available on the CDC Web site, Treating for Two, and using MothertoBaby fact sheets provided by the Organization of Teratology Information Specialists. A recent clinical opinion by Temming et al provides a practical approach to this decision-making process and includes a list of easily accessible on line resources. $^{31}$

- Women with chronic diseases requiring medication should have a preconception consultation that would allow a thorough discussion of the impact of their disease on pregnancy, as well as the risks and benefits of continuing a medication during pregnancy. When written resources are available, drug information should be linguistically appropriate.

- For women with complex medical conditions, the 6-week postpartum visit is an opportunity for transition of care including reassessment of medications and use of contraception if a medication is contraindicated in pregnancy or lactation.

\section{The Expert Panel's Suggested Research Gaps}

- Focused studies are needed to develop specific approaches for improved communication between providers and patients concerning the risks and benefits of the medications intended to be used recognizing that medication use during the perinatal period should be guided by shared decision making.

- The pharmacokinetics of most drugs used during pregnancy remains a major knowledge gap. Existing animal models need to be improved to address the mechanisms of actions of drugs and drug toxicity in pregnancy, while assessing clinical outcomes.

- Effective animal models and newer research techniques to study the use of medications during lactation are lacking. Research using cell lines, trophoblast tissue preparations, and placental perfusion models are useful to study placental transfer and metabolism. In addition, computational models may be useful for simulations and integration of data on fetal and placental compartments while "organ on a chip" models may be useful to further explore the contributions of the placenta and fetus.

- Specific information on medication efficacy is lacking due in part to limited accessibility to tissues where these medications act. Functional imaging methods are noninvasive tools that can provide functional and metabolic information in tissues in which medications act, which can help assess medication efficacy. Use of these methods needs to be incorporated in the study of medication use in the perinatal population.

- Drug safety in the pregnant women has been narrowly focused on the first-trimester exposure effects on the fetus leading to birth defects. However, many drugs may pose a risk when used inappropriately (or inadequately) while treating conditions that may adversely affect pregnancy outcomes. Thus, the safety issue has to be considered in a broader context beyond teratogenesis to develop appropriate dosage strategies taking into consideration the changing maternal physiology and chemical properties of the drug. More studies on all aspects of safety of medications in pregnancy and lactation are needed.

- It is also critical to study long- term outcomes beyond birth defects, which may include neurodevelopmental outcomes and functional impairments. This requires evaluating drug effects beyond "the most vulnerable" time in fetal development, recognizing that the brain continues to grow and mature beyond birth, and exposures during breastfeeding may need equal attention. Drug studies focused on lactation should extend beyond 6 months and must focus broadly to include both preterm and term neonates. There is an opportunity to collect more neonatal data on drugs by utilizing newer technologies on smaller specimens and has been successful in the neonatal drug network. ${ }^{29}$

\section{The Expert Panel's Policy-Related Considerations}

- Frequently, pregnancy is a contraindication for enrolling in clinical trials of new medications. However, many panel members noted that it is unethical to exclude pregnant women from drug trials, as the lack of critical data limits clinicians in making evidence-based decisions. Furthermore, inadequate or inappropriate medication use has the potential to increase maternal and fetal risks. These ethical and policy issues are summarized in recent publications. ${ }^{32,33}$ A systematic review by Coverdale et al that focused on randomized placebo-controlled trials of antidepressant medication given to pregnant women concluded that such trials were ethically justified. ${ }^{34}$ The argument-based approach emphasizes an ethical framework in which scientific studies should be following. The review endorsed the importance of conducting such trials as they would assist in appropriate treatment and build confidence in the physician's ability to prescribe antidepressant medication. Determining whether the impact on fetal development is due to the drug versus the mood disorder itself is equally important. ${ }^{34}$ Filling the knowledge gaps with well-designed studies could lead to new scientific knowledge to address the pressing needs of 
Table 1 Available safety monitoring systems

\begin{tabular}{|l|l|}
\hline Vaccine Adverse Event Reporting System (VAERS) & $\begin{array}{l}\text { A passive system whose data source includes patients, } \\
\text { health care providers, and manufacturers }\end{array}$ \\
\hline $\begin{array}{l}\text { Post-Licensure Rapid Immunization Safety Monitoring } \\
\text { (PRISM) }\end{array}$ & $\begin{array}{l}\text { Collaboration that links national health insurance plans, } \\
\text { immunizations registries, and other partners, e.g., } \\
\text { Centers for Medicare \& Medicaid Services }\end{array}$ \\
\hline Vaccine Safety Datalink (VSD) & $\begin{array}{l}\text { Collaboration between the Centers for Disease Control } \\
\text { and Prevention (CDC) and member health care } \\
\text { organizations }\end{array}$ \\
\hline Clinical Immunization Safety Assessment (CISA) Project & Collaboration of seven medical research centers \\
\hline Military Vaccine Agency & $\begin{array}{l}\text { Department of Defense program that focuses on bio- } \\
\text { logic threat preparedness, thus incorporating medica- } \\
\text { tions as well as vaccines }\end{array}$ \\
\hline VA Adverse Event Reporting System (ADERS) & VA surveillance system for vaccines and drugs \\
\hline Pregnancy Exposure Registries & $\begin{array}{l}\text { Passive system of specific drug exposures in pregnancy } \\
\text { (postmarket surveillance) }\end{array}$ \\
\hline $\begin{array}{l}\text { Food and Drug Administration (FDA) Adverse Events } \\
\text { Reporting System (FAERS) }\end{array}$ & $\begin{array}{l}\text { A postmarket safety surveillance of drugs and thera- } \\
\text { peutic biologic products }\end{array}$ \\
\hline FDA-Sentinel Program (after Mini-Sentinel Pilot) & $\begin{array}{l}\text { An active national electronic system to monitor safety } \\
\text { of FDA-regulated products }\end{array}$ \\
\hline $\begin{array}{l}\text { Vaccine and Medications in Pregnancy Surveillance } \\
\text { System (VAMPSS) }\end{array}$ & $\begin{array}{l}\text { Nationwide postmarket surveillance system that is } \\
\text { supported by public and private partnership }\end{array}$ \\
\hline
\end{tabular}

pregnant women and the intricacies related to medication use. $^{35}$

- Safety of medications administered during pregnancy is a major concern for clinicians and patients. Utilizing the robust surveillance system created for vaccines, which is continuously improving, provides an excellent model and starting point for drugs as well. This safety system identifies and evaluates adverse events with a combination of systems and partnerships (- Table 1). ${ }^{36}$

- The Vaccine and Medications in Pregnancy Surveillance System (VAMPSS), a postmarket surveillance system for vaccines and drugs, conducts case control and prospective cohort studies on certain drugs and vaccines in pregnancy. ${ }^{37}$ Birth defects are the main outcomes for these studies, and there is currently a need to broaden assessment to include long-term effects of these small numbers of drugs (asthma medications) and vaccines (influenza and Tdap).

Both the FDA and Congress attempted to improve appropriate pediatric drug information and labeling through a series of legislative activities that included the 1994 Pediatric rule, the 1997 FED Modernization Act(FDAMA), the 1998 Pediatric rule, the 2002 Best Pharmaceuticals for Children Act (BPCA), and the 2003 Pediatric Research Equity Act (PREA). Lessons learned from pediatric legislations suggest that similar obstetric equivalents of PREA and BPCA could be valuable. ${ }^{38,39}$

Until the "future" state is reached, enhancements to our current system can lead to important changes in data collection, which will improve clinical care for mothers and babies. Collaboration between federal agencies around implementation of safety system enhancements and existing data sources that could include pregnancy is needed. The obstetric aca- demic community and professional societies may collaborate as liaisons to federal entities for topics dealing with research, practice, and surveillance of drugs used during pregnancy and lactation.

In 2013, the CDC convened an expert group to develop a systematic approach to safer medications use during pregnancy. This expert group proposed a flow diagram to support expert review of available evidence on certain medications to provide consumers and clinicians with vetted information to decide whether to use a given drug in pregnancy. ${ }^{40}$ That proposed system has yet to be realized, and this most recent workshop further outlines several critical needs in the areas of clinical decision making, research, and policy. Pediatric clinicians recognized for decades that data on safety and efficacy of drugs in pediatrics was lacking for similar reasons outlined previously where pediatric medication data was extrapolated from adult studies. Their legislative experience and focus on children being included in research have led to vast improvements in this unique population. Similar efforts to improve the safety and efficacy of drugs for pregnant and lactating women are critically important now given this growing unique population.

Note

Workshop occurred at the Annual Meeting of the Society for Maternal-Fetal Medicine in February 2015 in San Diego, California.

Expert panelists:

Richard Beigi, MD, MSc-Magee-Women's Hospital of UPMC 
David Burchfield, MD-COFN/AAP

Cheryl Broussard, PhD-CDC

Alison Cahill, MD-Washington University School of Medicine

Steve Caritis, MD-Magee-Women's Hospital of UPMC

Christina Chambers, MD-UC San Diego School of Medicine

Shannon M. Clark, MD-University of Texas Medical Branch

Michael Cohen-Wolkoweiz, MD-Duke University Medical Center

Maged Milad Costantine, MD-University of Texas Medical Branch

Jonathan Davis, MD-Floating Hospital for Children at Tufts Medical Center

Maisa Feghali, MD-University of Pittsburgh

Michael F. Greene, MD-Massachusetts General Hospital Thomas W. Hale, RPh, PhD-Texas Tech University Health Sciences Center

Susan McCune, MD, MA Ed-FDA

Mirjana Nesin, MD-DMID/NIAID/NIH

Tonse Raju, MD-NIH

Sonja Rasmussen, MD-CDC

Adelaide Robb, MD-Children's National Medical Center George Saade, MD-University of Texas Medical Branch

Melissa S. Tassinari, PhD, DABT-FDA

Barbara Wesley, MD, MPH-FDA

Amina White, PhD-NIH, Department of Bioethics

Chris Zahn, MD-ACOG

Anne Zajicek, MD, PharmD, FAAP-NICHD

\section{Conflict of Interest}

None.

\section{References}

1 Kuklina E, Callaghan W. Chronic heart disease and severe obstetric morbidity among hospitalisations for pregnancy in the USA: 1995-2006. BJOG 2011;118(3):345-352

2 Campbell KH, Savitz D, Werner EF, et al. Maternal morbidity and risk of death at delivery hospitalization. Obstet Gynecol 2013; 122(3):627-633

3 Small MJ, James AH, Kershaw T, Thames B, Gunatilake R, Brown H. Near-miss maternal mortality: cardiac dysfunction as the principal cause of obstetric intensive care unit admissions. Obstet Gynecol 2012;119(2 Pt 1):250-255

4 Kaaja RJ, Greer IA. Manifestations of chronic disease during pregnancy. JAMA 2005;294(21):2751-2757

5 Bateman BT, Bansil P, Hernandez-Diaz S, Mhyre JM, Callaghan WM, Kuklina EV. Prevalence, trends, and outcomes of chronic hypertension: a nationwide sample of delivery admissions. Am J Obstet Gynecol 2012;206(2):134.e1-134.e8

6 Farr SL, Hayes DK, Bitsko RH, Bansil P, Dietz PM. Depression, diabetes, and chronic disease risk factors among US women of reproductive age. Prev Chronic Dis 2011;8(6):A119-A119

7 Rooney BL, Schauberger CW, Mathiason MA. Impact of perinatal weight change on long-term obesity and obesity-related illnesses. Obstet Gynecol 2005;106(6):1349-1356

8 Tyer-Viola LA, Lopez RP. Pregnancy with chronic illness. J Obstet Gynecol Neonatal Nurs 2014;43(1):25-37
9 Dunlop AL, Jack BW, Bottalico JN, et al. The clinical content of preconception care: women with chronic medical conditions. Am J Obstet Gynecol 2008;199(6, Suppl 2):S310-S327

10 Josephson MA, McKay DB. Pregnancy and kidney transplantation. Semin Nephrol 2011;31(1):100-110

11 Mitchell AA, Gilboa SM, Werler MM, Kelley KE, Louik C, Hernández-Díaz S; National Birth Defects Prevention Study. Medication use during pregnancy, with particular focus on prescription drugs: 1976-2008. Am J Obstet Gynecol 2011; 205(1):51.e1-51.e8

12 Werler MM, Mitchell AA, Hernandez-Diaz S, Honein MA. Use of over-the-counter medications during pregnancy. Am J Obstet Gynecol 2005;193(3 Pt 1):771-777

13 American Academy of Pediatrics Committee on Drugs. Transfer of drugs and other chemicals into human milk. Pediatrics 2001; 108(3):776-789

14 Ryan AS, Wenjun Z, Acosta A. Breastfeeding continues to increase into the new millennium. Pediatrics 2002;110(6):1103-1109

15 Stultz EE, Stokes JL, Shaffer ML, Paul IM, Berlin CM. Extent of medication use in breastfeeding women. Breastfeed Med 2007; 2(3):145-151

16 Anderson PO, Pochop SL, Manoguerra AS. Adverse drug reactions in breastfed infants: less than imagined. Clin Pediatr (Phila) 2003; 42(4):325-340

17 Moore KL, Persaud TVN, Torchia MG. The Developing Human: Clinically Oriented Embryology. 10th ed. Philadelphia, PA: Elsevier; 2015

18 Feghali M, Venkataramanan R, Caritis S. Pharmacokinetics of drugs in pregnancy. Semin Perinatol 2015;39(7):512-519

19 Gabbe SG, Neibyl JR, Simpson LJ, et al. Obstetrics Normal and Problem Pregnancies. 6th ed. Philadelphia, PA: Elsevier Saunders; 2012

$20 \mathrm{Kim} \mathrm{JH}$, Scialli AR. Thalidomide: the tragedy of birth defects and the effective treatment of disease. Toxicol Sci 2011;122(1):1-6

21 Tabacova S, Little R, Tsong Y, Vega A, Kimmel CA. Adverse pregnancy outcomes associated with maternal enalapril antihypertensive treatment. Pharmacoepidemiol Drug Saf 2003;12(8):633-646

22 Cooper WO, Hernandez-Diaz S, Arbogast PG, et al. Major congenital malformations after first-trimester exposure to ACE inhibitors. N Engl J Med 2006;354(23):2443-2451

23 Briggs GG. Drug effects on the fetus and breast-fed infant. Clin Obstet Gynecol 2002;45(1):6-21

24 Sachs HC; Committee On Drugs. The transfer of drugs and therapeutics into human breast milk: an update on selected topics. Pediatrics 2013;132(3):e796-e809

25 Hale TW. Maternal medications during breastfeeding. Clin Obstet Gynecol 2004;47(3):696-711

26 Berlin CM Jr, Paul IM, Vesell ES. Safety issues of maternal drug therapy during breastfeeding. Clin Pharmacol Ther 2009;85(1):20-22

27 Stover MW, Davis JM. Opioids in pregnancy and neonatal abstinence syndrome. Semin Perinatol 2015;39(7):561-565

28 Chambers C. Over-the-counter medications: risk and safety in pregnancy. Semin Perinatol 2015;39(7):541-544

29 Clark SM, Dunn HE, Hankins GD. A review of oral labetalol and nifedipine in mild to moderate hypertension in pregnancy. Semin Perinatol 2015;39(7):548-555

30 Beigi RH. The importance of studying antimicrobials in pregnancy. Semin Perinatol 2015;39(7):556-560

31 Temming LA, Cahill AG, Riley LE. Clinical management of medications in pregnancy and lactation. Am J Obstet Gynecol 2016; 214(6):698-702

32 Zimmerman K, Gonzalez D, Swamy GK, Cohen-Wolkowiez M. Pharmacologic studies in vulnerable populations: using the pediatric experience. Semin Perinatol 2015;39(7):532-536

33 White A. Accelerating the paradigm shift toward inclusion of pregnant women in drug research: ethical and regulatory considerations. Semin Perinatol 2015;39(7):537-540 
832 Improving Safe and Effective Use of Drugs in Pregnancy and Lactation Riley et al.

34 Coverdale JH, McCullough LB, Chervenak FA. The ethics of randomized placebo-controlled trials of antidepressants with pregnant women: a systematic review. Obstet Gynecol 2008;112(6):1361-1368

35 Blehar MC, Spong C, Grady C, Goldkind SF, Sahin L, Clayton JA. Enrolling pregnant women: issues in clinical research. Womens Health Issues 2013;23(1):e39-e45

36 Nesin M, Sparer O. Vaccine monitoring systems: a potential model for medications in pregnancy. Semin Perinatol 2015;39(7):524-529

37 American Academy of Allergy, Asthma \& Immunology website. The Vaccines and Medications in Pregnancy Surveillance System (VAMPSS). http://www.aaaai.org/about-aaaai/strategic-relationships/vampss. Accessed December 19, 2016
38 Ren Z, Zajicek A. Review of the Best Pharmaceuticals for Children Act and the Pediatric Research Equity Act: what can the obstetric community learn from the pediatric experience? Semin Perinatol 2015;39(7):530-531

39 Institute of Medicine. Addressing the Barriers to Pediatric Drug Development: Workshop Summary. Washington, DC: The National Academies Press; 2008

40 Broussard CS, Frey MT, Hernandez-Diaz S, et al. Developing a systematic approach to safer medication use during pregnancy: summary of a Centers for Disease Control and Preventionconvened meeting. Am J Obstet Gynecol 2014;211(3): 208-214.e1 